\title{
OBTENCIÓN DE LAS BENTONITAS A PARTIR DE LAS TOBAS
}

Silvio Paz Chino ${ }^{1}$

José Valdez Aduviri ${ }^{2}$

\author{
R E S U M E N
}

Se ha dado gran preferencia a la explotación de minerales metálicos, riqueza del subsuelo y el estudio crítico de las mismas bajo un aspecto geológico y minero; sin embargo, no sucede lo mismo con los yacimientos y minerales no metálicos a lo largo y ancho de nuestro país, especialmente en nuestra región.

Por estas razones nuestro objetivo es incentivar y propiciar el estudio y explotación de estas riquezas no metálicas, en forma racional y con una tecnología avanzadas para crear un polo de desarrollo en la región en base a estos elementos, especialmente la puzolana, que existe a lo largo del Valle de Tacna en inmensas cantidades.

\section{A B S TR A C T}

It has been given great preference to the exploitation of metallic minerals, wealth of the underground and the critical study of these in the view of geological and miner; however, the same thing doesn't happen with locations and non metallic minerals to the long and wide of our country, especially in our region.

For this reason our objective is to incentivate and to propitiate the study and exploitation of these non metallic wealth, in rational form and with an advanced technology to create a development pole in the region based on these elements, especially the puzolana that exists along the Valley of Tacna in large quantities.

\section{INTRODUCCIÓN}

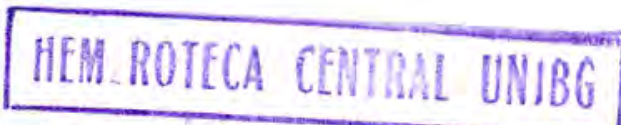

Los minerales no metálicos son las materias primas más utilizadas en las diferentes industrias, desde una antigüedad que se remonta hasta el año 4500 A.C.

Sin embargo, el uso incipiente de estas materias primas no metálicas ha dado origen al uso no tecnológico y por tanto es poco el conocimiento que se tiene de ellos.

En el Perú, a diferencia de otros países desarrollados, la explotación de materiales no metálicos es muy incierta pese acontarcon ingentes cantidades, tales como carbón, piedras ornamentales, arcillas en diferentes clases, baritina, ónix, mármol, etc.

En la región sur de nuestro país tenemos la fortuna de contar con inmensos yacimientos de minerales metálicos y no metálicos, especialmente de este último, desarrollar una explotación nacional y con tecnología adecuada daría a nuestra región un polo de desarrollo inmenso, dada la ubicación estratégica del Departamento de Tacna. 
Los autores de este trabajo sobre las arcillas provenientes de las tobas o cenizas volcánicas han considerado oportuno hacer los primeros ensayos tanto en laboratorio como en el campo para determinar la posibilidad de su explotación a escala industrial.

Nuestra hipótesis sostiene que como componente de las cenizas volcánicas estaría la "Bentonita", material de mucha demanda en la industria, dadas - sus especiales características.

\section{CARACTERÍSTICAS DEL YACIMIENTO}

Las cenizas volcánicas, se ha dicho, son compuestos considerados como silicatos de aluminio amorfo y cristalino mezclados con magnesio, fierro, calcio y otros elementos alcalinos.

\section{TOBAS VOLCÁNICAS EN LA REGIÓN DE TACNA}

La serie sedimentaria que contiene estrato de tufo dacítico con propiedades puzolánicas, pertenece estratigráficamente a la formación Maure, con características lenticulares en sus estratos, observándose variaciones laterales en el espesor como en el color de las mismas.

Litológicamente la ceniza volcánica, consiste en un polvo suelto de color blanco o rosado con fragmentos de pómez blanco y cristalinos de cuarzo bipiramidal, estos depósitos carecen completamente de estratificación, y se presentan como una masa homogénea sin estructuras, pero a cierta distancia debajo de la superficie, el depósito se presenta más compacto y con la textura de un tufo.

Como guía geomorfológica puede anotarse que el material no metálico aflora en flancos de los pliegues, que son lunares donde han sido ubicados los horizontes productivos (en las zonas de Alto Pachía).

\section{DEPÓSITO DE LA CENIZA VOLCÁNICA EN LA ZONA DE ESTUDIO}

Ciertas zonas de la región Pachía-Palca, contienen depósitos de ceniza y tufo volcánico que forman un manto delgado y discontinuo encima de las terrazas y depósitos de piedemonte. Los depósitos mayores de cenizas afloran en los cerrillos blancos de la esquina sur-occidental, del cuadrángulo de Pachía y Uchusuma. En la parte media del Valle del Caplina, las cenizas se encuentran como fajas angostas a lo largo del valle y aproximadamente a $50 \mathrm{~m}$ sobre el nivel del río.

Litológicamente la ceniza volcánica consiste en polvo suelto de color blanco o rosado con fragmentos de pómez blanco y cristalitos de cuarzo bipiramidal. Estos depósitos carecen completamente de estratificación y se presentan con una masa homogénea, sin estructura, pero a cierta distancia debajo de la superficie, el depósito se presenta más compacto y con la textura de un tufo.

Las cenizas sobreyacen a terrazas aluviales y depósitos de piedemonte, los cuales, probablemente, son más o menos coetáneos con los depósitos fluvioglaciares superyacentes a la formación Maure. Por lo tanto pese a las similitudes litológicas, los depósitos de cenizas son más jóvenes que los piroclásticos de la formación Maure. Se ha concluido que el contenido de cuarzo bipiramidal en ambas rocas piroclásticas no tienen ningún valor para correlación.

\section{ENSAYO DE GRANULOMETRIA}

Ver cuadro 1 de ensayo de granulometria y gráfico.

\section{DISEÑO DE PLANTA DE SEPARACIÓN}

El diseño para procesar consiste en un lavador y clasificador de la ceniza. Esta instalación comprende una tolva con parrilla inclinada con agujeros para el paso del agua, otro depósito con una criba incorporada, seguida por una tolva cónica para la recepción del líquido y una bomba de agua para alimentación del líquido elemento desde un tanque, además otro tanque para los sedimentos de arcilla (ver figura 1).

El lavador clasificador de la ceniza comprende: una parilla inclinada, sobre la cual vuelca el flujo de pulpa; tobas volcánicas, una turbina que impele una corriente de agua adicional atravezando la parrilla de abajo hacia arriba para arrastrar a los elementos finos y la piedra pómez, mientras que la arena gruesa se desliza a lo largo de la parrilla y cae en un conducto de recuperación.

La mezcla de ceniza fina más la piedra pómez dirigida a una criba vibradora de malla muy fina; los rechazos de la criba (piedra pómez, arena fina) son seleccionados, y el material que pasa eseliminado con el agua (arcilla).

El reglaje del tamaño de separación se hace actuando sobre la velocidad de corriente de agua adicional. 
Cuadro 1. Ensayo de granulometría.

Laboratorio de físico química.

Proyecto :
Fecha $:$ Bentonita"

\begin{tabular}{|c|c|c|c|c|c|c|c|c|}
\hline \multicolumn{2}{|c|}{ MALLAS } & \multicolumn{2}{c|}{ RETENIDO } & \multicolumn{2}{c|}{$\%$ PESO } & \multicolumn{3}{|c|}{$\begin{array}{c}\text { ESPECIFI- } \\
\text { CACIONES }\end{array}$} \\
\hline $\begin{array}{c}\text { Serie } \\
\text { Amé- } \\
\text { rica }\end{array}$ & $\begin{array}{c}\text { Abert } \\
\text { En } \\
\text { mm }\end{array}$ & $\begin{array}{c}\text { gra- } \\
\text { mos }\end{array}$ & $\begin{array}{c}\% \\
\text { Par- } \\
\text { cial }\end{array}$ & $\begin{array}{c}\text { Ret. } \\
\text { T }\end{array}$ & $\begin{array}{c}\text { Peso } \\
\text { T }\end{array}$ & & $\begin{array}{c}\text { Pe- } \\
\text { so }\end{array}$ & \\
\hline $2^{\prime \prime}$ & 50.00 & & & & & & & \\
\hline $11 / 2^{\prime \prime}$ & 37.50 & & & & & & & \\
\hline $1^{\prime \prime}$ & 25.00 & & & & & & & \\
\hline $3 / 4^{\prime \prime}$ & 19.00 & & & & & & & \\
\hline $1 / 2^{\prime \prime}$ & 12.50 & & & & & & & \\
\hline $3 / 8^{\prime \prime}$ & 9.50 & & & & & & & \\
\hline
\end{tabular}

\begin{tabular}{|c|r|l|l|l|l|l|l|l|}
\hline $3 / 8^{\prime \prime}$ & 9.50 & & & & & & & \\
\hline $1 / 4^{\prime \prime}$ & 6.68 & & & & & & & \\
\hline No.4 & 4.75 & & & & 100.00 & & & \\
\hline
\end{tabular}

\begin{tabular}{|c|r|l|l|l|l|l|l|l|}
\hline No.4 & 4.75 & & & & 100.00 & & & \\
\hline$* 6$ & 3.360 & & & & & & & \\
\hline$" 8$ & 2.38 & 16.65 & 3.33 & 3.33 & 96.67 & & & \\
\hline$* 10$ & 2.00 & & & & & & & \\
\hline
\end{tabular}

\begin{tabular}{|l|r|r|r|r|r|l|l|l|}
\hline$* 10$ & 2.00 & & & & & & & \\
\hline$* 16$ & 1.18 & 36.82 & 7.37 & 10.7 & 89.30 & & & \\
\hline$" 20$ & 0.85 & & & & & & & \\
\hline
\end{tabular}

\begin{tabular}{|r|r|r|l|l|l|l|l|l|l|}
\hline$" 20$ & 0.85 & & & & & & & \\
\hline$" 30$ & 0.60 & 60.85 & 12.17 & 22.87 & 77.13 & & & \\
\hline$" 40$ & 0.425 & & & & & & & \\
\hline$" 50$ & 0.300 & 57.61 & 11.52 & 34.39 & 65.61 & & & \\
\hline
\end{tabular}

\begin{tabular}{|r|r|r|r|r|r|l|l|l|}
\hline$" 80$ & 0.177 & & & & & & & \\
\hline$" 100$ & 0.150 & 74.40 & 14.88 & 49.27 & 50.73 & & & \\
\hline$" 200$ & 0.075 & 65.00 & 13.00 & 62.27 & 37.73 & & & \\
\hline$" 200$ & & $\begin{array}{r}188 .- \\
67\end{array}$ & 37.73 & $\begin{array}{r}100 .- \\
00\end{array}$ & & & & \\
\hline
\end{tabular}

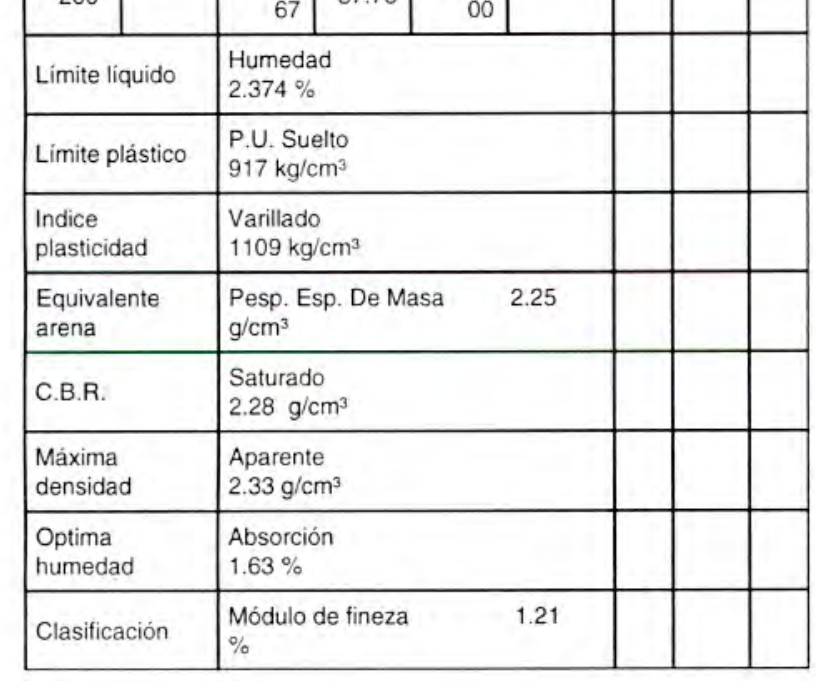

\section{SILICE GRANULADA (ELEMENTO PESADO).}

En la recuperación de los elementos pesados, la sílice es una de las sustancias más extendidas, a tal punto que una gran parte de la corteza terrestre está formada por compuestos de silicio, este se encuentra como silicio (IV) óxido $\left(\mathrm{SiO}_{2}\right)$, llamado también óxido de silicio, en distintas formas mineralógicas como: cuarzo, arena, cristal de roca o como silicatos.

La sílice tratada cuya pureza alcanza el $58 \%$ puede usarse en el revestimiento de paredes, fabricación de vidrio, etc.

\section{Piedra Pómez}

Los elementos flotantes, o la piedra pómez, es un mineral esponjoso y frágil, procedente de las emanaciones volcánicas, cuyo peso es menor que el agua por sus numerosos poros, alcanza a un $34 \%$.

\section{Arcilla (elemento fino)}

La arcilla es un material muy fino que sirve para manufacturar material refractario y cerámico, alcanza un $7 \%$.

\section{Recuperación de las aguas cargadas}

La evacuación de las aguas de desbordamiento de los clasificadores está reglamentada por la legislación vigente para evitar la polución de la corriente de agua.

Como la cantera no dispone de suficiente agua, se recupera las aguas usadas, después de la eliminación de los materiales en suspensión.

La solución más adecuada consiste en usar el tanque de decantación, que es una cuba de hormigón de gran superficie y poca profundidad, a la cual se envía las aguas cargadas para su decantación. El agua desembarazada con los elementos en suspensión es aspirado a un depósito, y reciclada. Habitualmente se utiliza dos depósitos uno en servicio y otro en curso de decantación para ulterior evacuación de los lodos. La tabla muestra el resultado promedio de análisis de tratamiento de la ceniza volcánica: 


\begin{tabular}{|l|c|c|}
\hline \multicolumn{1}{|c|}{ COMPONENTES } & GRAMOS & $\%$ \\
\hline Silice & 480 & 48 \\
Piedra pómez & 370 & 37 \\
Arcilla & 150 & 15 \\
\hline TOTAL & 1000 & 100 \\
\hline
\end{tabular}

\section{BENTONITA}

\section{ANTECEDENTES}

Según Elisa Leoniaz, las bentonitas son tobas volcánicas alteradas e hidrolizadas que se caracterizan por su hinchamiento, por lo general bentonitas de sodio $(\mathrm{Na})$ consideradas como de tipo I y el de tipo II Bentonitas que no hinchan por la presencia de calcio (Ca). Tanto el sodio como el calcio aparecen como cationes principales. Algunas veces por su contenido de calcio (tipo II) se llaman Esmectitas que son rocas arcillosas compactas de colores blanco, gris, hasta rosado que aparecen en capas de potencia variable y que al meteorizarse son friables. En contacto con el agua se desmorona con facilidad.

La bentonita propiamente dicha es una roca análoga a la arcilla de color gris a blanco amarillento.

Si se introduce en agua se hincha aumentando su volumen hasta alcanzar unas 6 o más veces su volumen, inicial, formando un gel tenaz, en ello estriba su valor técnico aplicado en la industria.

El componente principal de la ESMECTITA y Bentonita es la Montmorillonita cuya capacidad para hincharse estriba en su red cristalina con moléculas de $\mathrm{SiO}_{2}$ y $_{2} \mathrm{O}$.

Es muy importante señalar que varios autores afirman que las bentonitas yacen en series marinas, lacustres (como el de Ayacucho), mientras que en Europa Mediterránea en zonas volcánicas (podría ser lo mismo que en Tacna).

W. Siegel definitivamente relaciona la bentonita con el vidrio volcánico, químicamente existen relaciones con el volcanismo residual, cuyos componentes químicos (según Senger Félix), son:

\section{Bentonita de Florida USA}

\begin{tabular}{|l|r|}
\hline Sustancia & $\%$ \\
\hline $\mathrm{SiO}_{2}$ & 55.28 \\
$\mathrm{Al}_{2} \mathrm{O}_{3}$ & 21.90 \\
$\mathrm{Fe}_{2} \mathrm{O}_{3}$ & 2.66 \\
$\mathrm{~K}_{2} \mathrm{O}$ & 6.21 \\
$\mathrm{Na}_{2} \mathrm{O}$ & 5.10 \\
$\mathrm{TiO}_{2}$ & 0.28 \\
$\mathrm{CaO}$ & 1.88 \\
$\mathrm{MgO}$ & 0.37 \\
$\mathrm{H}_{2} \mathrm{O}$ & 5.64 \\
$\mathrm{OtroS}$ & 0.68 \\
\hline & 100.00 \\
\hline
\end{tabular}

Destacados autores internacionales afirman que la Bentonita es originada por la descomposición de ceniza volcánica como la que se confirmaría en la zona de Tacna como producto de lavas volcánicas.

La bentonita es altamente plástica y fina de modo que con una clasificación (granulométrica) cuidadosa se podría obtener para la industria un producto de alta calidad.

La bentonita por su propiedad de hincharse por su mayor contenido de sodio ( $\mathrm{Na}$ ) como cualquier otra familia de las arcillas puede ser graficada en base a esta característica (absorción de agua) según se aprecia en la curva de absorción de las arcillas.

Curva de Absorción de las Arcillas.

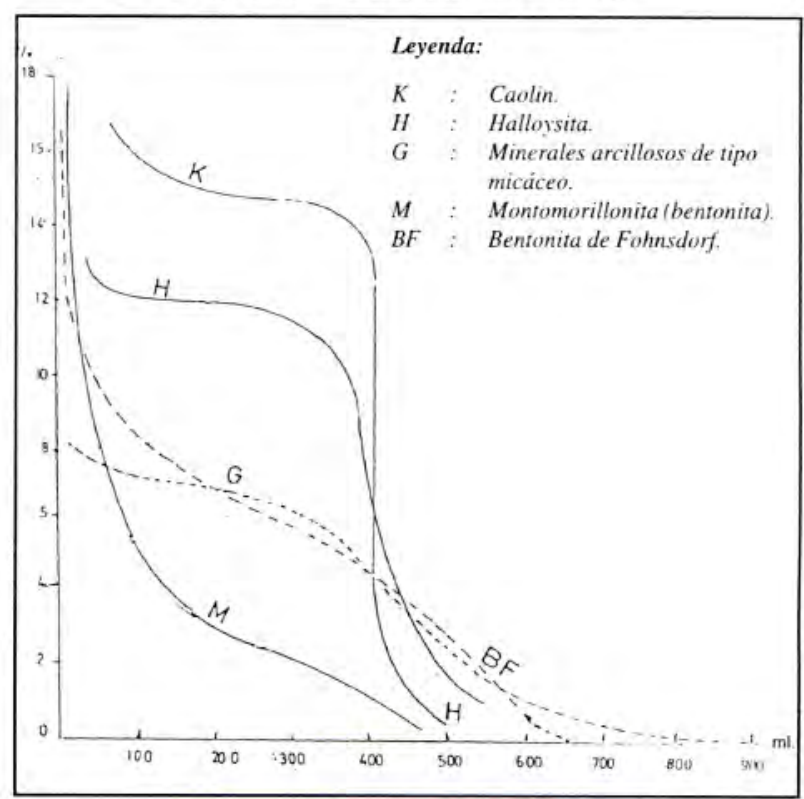


ANÁLISIS QUÍMICO DE LAS BENTONITAS EN LAZONA DE TACNA

\section{Bentonita de la zona de Tacna}

\begin{tabular}{|l|r|}
\hline Sustancia & $\%$ \\
\hline $\mathrm{SiO}_{2}$ & 54.62 \\
$\mathrm{Al}_{2} \mathrm{O}_{3}$ & 21.80 \\
$\mathrm{Fe}_{2} \mathrm{O}_{3}$ & 2.00 \\
$\mathrm{~K}_{2} \mathrm{O}$ & 3.42 \\
$\mathrm{NaO}$ & 15.40 \\
$\mathrm{CaO}$ & 0.40 \\
$\mathrm{MgO}$ & 2.48 \\
Otros & 0.55 \\
\hline & 100.00 \\
\hline
\end{tabular}

\section{Aplicaciones de la bentonita en la industria}

Son muy variados:

- En principio, son muy requeridas para desengrasar lana, en las textilerías, de ahí su nombre "tierra de batán".

- Es requerido para la decoloración de todo tipo de aceites después de haber sido secados a más de $1000^{\circ} \mathrm{C}$.

- Mediante activación y tratamiento con álcalis se fabrica un compuesto llamado "Thixotona" que se hincha considerablemente en agua y que una vez licuado por agitación, vuelve rápidamente a formar un gel viscoso cuando se deja de agitar. Esta propiedad es conocida con el nombre de Tixitropia, es de importancia para la técnica de sondeos con lodos densos, especialmente utilizados en sondeos petrolíferos.

La bentonita que se esponja es utilizada en exploraciones y explotaciones petroliferas en USA.

- La capacidad de hinchamiento de la Bentonita es también aprovechada en la industria de la cerámica en la fabricación de arenas de moldeo, en la de crisoles y como medio de aumentar la plasticidad en la industria de cerámica.

- Se emplea en la fabricación de pinturas conductores eléctricos.

\section{CONCLUSIONES}

- En un trabajo de investigación sobre las tobas volcánicas o cenizas volcánicas aplicadas a materiales de construcción antisismica, se determinó que el material fino obtenido daba el comportamiento y características de una bentonita una variedad de las arcillas.

- La bentonita y el azufre mezclados en un volumen de $60 \%$ a $40 \%$ respectivamente y puesto a una temperatura en ebullición de 100 a $115^{\circ} \mathrm{C}$ da una masa viscosa que ha sido utilizada en laboratorio de mecánica de rocas, como recubrimiento de las probetas de concreto para uniformizar sus caras y llevar a cabo el ensayo de esfuerzo a la compresión (resistencia a la rotura). Esta bentonita así considerada ha sido obtenida de las cenizas volcánicas.

- El uso de la posible bentonita y dado su comportamiento fue obtenido como material fino de las cenizas volcánicas, y de las diferentes canteras que se encuentran en el valle de Tacna (zona de Chorrillos y Calana).

- Un estudio físico-mecánico y bibliográfico utilizado en el presente trabajo ha determinado en una primera fase, que el material fino obtenido (malla $50 \mathrm{~mm}$ ) es una variedad de las arcillas, lo que no necesariamente debe tratarse como una "bentonita".

- Procediendo con los análisis químicos el material fino en el laboratorio de físico química, los resultados han demostrado que no se trataría de una "bentonita" debido a diferencias en sus elementos constituyentes. El principal componente de la "bentonita" es la montmorillonita, cuyos elementos químicos la representan definitivamente como una variedad de las arcillas.

Los cuadros siguientes muestran estas diferencias:

\begin{tabular}{|l|r|}
\hline \multicolumn{2}{|l|}{$\begin{array}{l}\text { Arcilla común (material fino obtenido de las cenizas } \\
\text { volcánicas) }\end{array}$} \\
\hline Sustancia & $\%$ \\
\hline $\mathrm{SiO}_{2}$ & 54.62 \\
$\mathrm{Al}_{2} \mathrm{O}_{3}$ & 21.80 \\
$\mathrm{Fe}_{2} \mathrm{O}_{3}$ & 2.00 \\
$\mathrm{~K}_{2} \mathrm{O}$ & 3.42 \\
$\mathrm{NaO}$ & 15.40 \\
$\mathrm{CaO}$ & 0.04 \\
$\mathrm{MgO}$ & 2.48 \\
Otros & 0.55 \\
\hline & 100.00 \\
\hline
\end{tabular}




\begin{tabular}{|l|r|}
\hline \multicolumn{2}{|l|}{ Componente de la Bentonita (Montmorillonita) } \\
\hline Sustancia & $\%$ \\
\hline $\mathrm{SiO}_{2}$ & 55.28 \\
$\mathrm{Al}_{2} \mathrm{O}_{3}$ & 21.90 \\
$\mathrm{Fe}_{2} \mathrm{O}_{3}$ & 2.66 \\
$\mathrm{~K}_{2} \mathrm{O}$ & 6.21 \\
$\mathrm{Na}_{2} \mathrm{O}$ & 5.10 \\
$\mathrm{CaO}$ & 1.88 \\
$\mathrm{MgO}$ & 0.37 \\
$\mathrm{H}_{2} \mathrm{O}$ & 5.64 \\
$\mathrm{Otros}$ & 0.68 \\
\hline & 100.00 \\
\hline
\end{tabular}

- Sin embargo, a través de estudios y análisis de sus componentes en laboratorio, definitvamente no co-rresponde a la bentonita; en cambio se determinó un tipo de arcilla muy rica en óxidos de silice y aluminio y otros como magnesio, sodio, potasio y fierro, etc. que determinado en sus porcentajes, sus componentes con estudios más detallados, estariamos en condiciones de tipificar el tipo de arcilla y poder industrializar en base a sus características.

- Pese a la opinión de estudiosos sobre el origen de las bentonitas, como producto de derrames volcá-

\section{REFERENCIAS BIBLIOGRÁFICAS}

BERNARD LEACH, "A potters book", London 1977.

III Simposium de Minería 1989.

PETER STARKEY, "Saltglaze", London 1977.

PETER HALD, "Técnica de cerámica”, Barcelona 1973. nicos, el análisis químico en laboratorio confirma que se trata solo de arcilla común y no de la "bentonita" como se ha demostrado en los cuadros anteriores (diferencia de constituyentes).

- Para confirmar si las muestras obtenidas de las cenizas volcánicas son "Bentonitas" o no, se debe acudir a la preparación de una mezcla de material fino, obtenido por proceso mecánico, agregando a esta mezcla el reactivo bencidina en presencia de cloruro férrico diluido. Esta mezcla pastosa debe dar una coloración azul. La prueba no se realizó por no contar con el reactivo bencidina, dado su carácter cancerígeno y a la vez costoso

- Descartado que el material fino obtenido de las cenizas volcánicas correspondan a las bentonitas, hemos continuado con los estudios y análisis para determinar el tipo de arcillas y variedad de las tobas volcánicas.

- Por otra parte, la plasticidad mostrada en los ensayos de laboratorio nos satisfacen las normas existentes para este tipo de material, debido a la irregular toma de muestras de campo, por lo que es imprescindible continuar con la determinación correcta del tipo de suelos y el yacimiento en estudio.

REVISTAS. varias.

THOMAS SHAFER, "Pottery decoration" New York 1976.

W.E. PETRASHECK, "Yacimientos y criaderos"..

Figura 1. Proyecto (Bentonita)

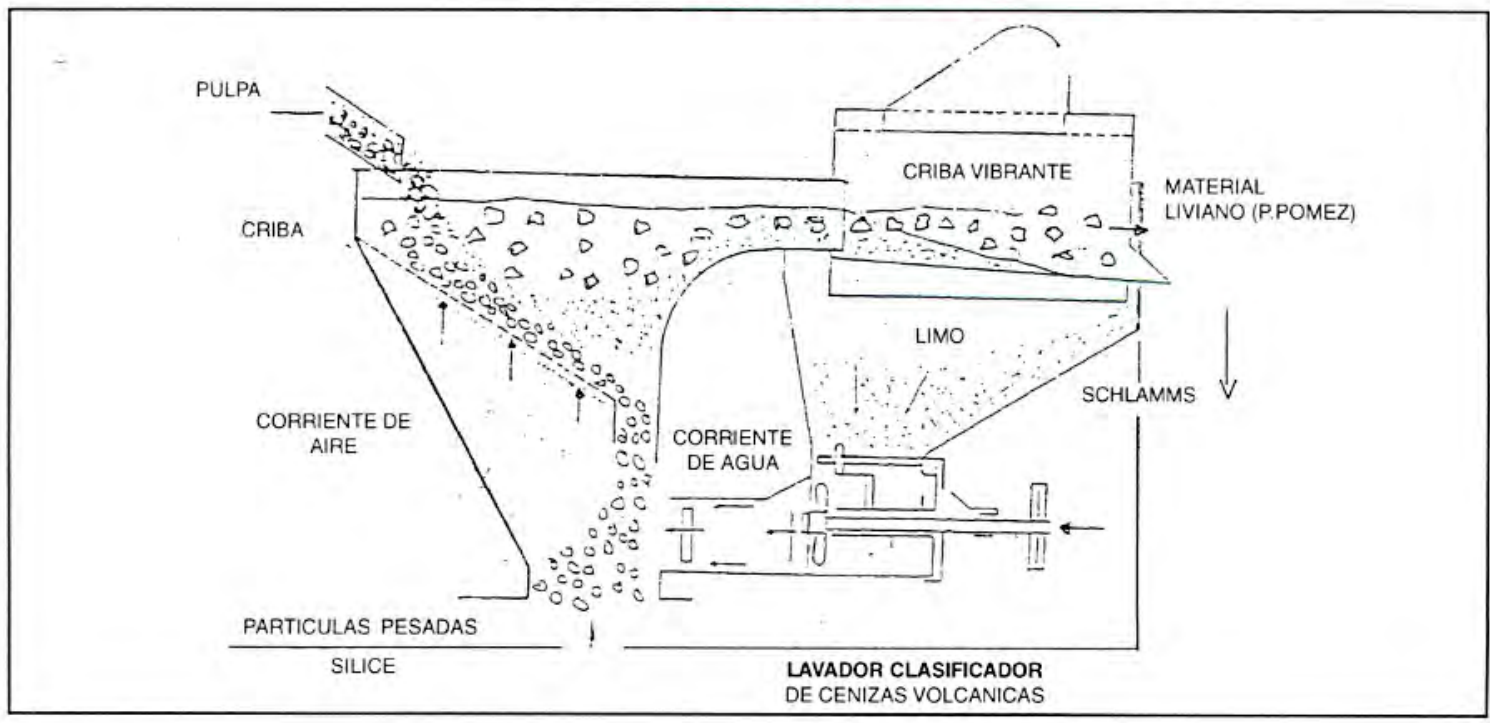

